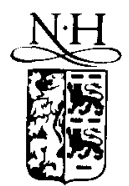

ELSEVIER

\title{
Evaluation of the probing profile of scanning force microscopy tips
}

\author{
S.S. Sheiko ${ }^{*, a, 1}$, M. Möller ${ }^{*, a, 1}$, E.M.C.M. Reuvekamp ${ }^{\text {b }}$, H.W. Zandbergen ${ }^{\text {c }}$ \\ CMO-Center for Materials Research, ${ }^{a}$ Department of Chemical Technology and ${ }^{b}$ Department of Applied Physics, \\ University of Twente, P.O. Box 217, 7500 AE Enschede, The Netherlands \\ ${ }^{c}$ National Center for HREM, Delft University of Technology, Rotterdamseweg 137, 2628 AL Delft, The Netherlands
}

(Received 4 May 1993; in final form 26 October 1993)

\begin{abstract}
It is demonstrated that a high-temperature-treated (305) surface of a $\mathrm{SrTiO}_{3}$ crystal can be used to evaluate the probing profile of AFM tips routinely, to provide a means of selecting perfect tips and to evaluate possible image distortions. This is important in order to recognize typical AFM artifacts which are caused by tips with truncated or twinned peaks which occur rather often in the case of microfabricated AFM needles. By means of selected needles, it is shown that also defective tips can give apparently rather perfect atomic resolution from flat crystal surfaces. Scope and limitations of the resolution of structural defects are discussed as the criterion for real atomic resolution.
\end{abstract}

\section{Introduction}

Since the introduction of Atomic Force Microscopy (AFM) [1], the applications of this method are rapidly growing in various fields of materials science [2-5]. This is based on unique possibilities and advantages in comparison with other microscopic techniques, e.g. 3D surface imaging down to the atomic scale, ambient and variable measuring conditions, and last but not least the relatively simple sample preparation. However, the reliability of AFM data is often questionable due to the lack of insight into the

\footnotetext{
* Corresponding author.

1 Present address: Organische Chemie III/Makromolekulare Chemie, Universität Ulm, D-89069 Ulm, Germany.
}

actual imaging process, and the recognition of artifacts represents an issue of current research activities. Most artifacts and many limitations are caused by two effects: (i) the superposition of the shape and structure of the probing tip on the image of the investigated surface, (ii) the inelastic deformation of a sample surface induced by the AFM tip [6-9].

Tip-shape-dependent artifacts can be observed at the level of micrometers, nanometers (mesoscopic) and also on the atomic scale. Typically, micrometer-scale artifacts are tip images caused by elevated surface structures which are steeper than the tip itself $[6,7]$. These artifacts are relatively easy to be recognized due to their prominent appearance as pyramids or triangular-shaped patterns. Supplementary scanning electron microscopy measurements can be done to verify the AFM micrographs. 
Also AFM images of regular structures at the atomic scale depend on the shape and properties of the probing tip. Key parameters are the shape of the tip, the contact area, the modulus and the applied force [10]. In many cases, atomic images can be scrutinized by X-ray or TEM investigations and computer simulations which give detailed information about the crystalline structure. However, so far it is not possible to evaluate and verify local defects. AFM artifacts on the mesoscopic ${ }^{\# 1}$ level can often not be recognized and evaluated. Convolution of the tip shape with the actual surface structure of the sample results in "mesorealistic" pictures which are mixed images of the tip and the sample surface. Especially with organic materials, scanning electron microscopy is often useless for comparison because the images do not provide sufficient resolution or suffer from electron radiation damage. Both qualitative and quantitative information such as shape, roughness and size distribution taken from the mesoscopic level are questionable as long we cannot rely on methods for the mesoscopic control of the AFM tip.

Thus, from practical experience and theoretical considerations, the exact determination of the shape and the structure of the probing site of an AFM tip is essential in order to evaluate AFM images for the actual size and real shape of the visualized structures and to recognize artifacts unambiguously and easily.

In the present paper we discuss a selection of typical AFM artifacts and describe a simple and reliable method to evaluate the mesoscopic profile of a probing tip. Although the development of AFM tips with high aspect ratio and improved sharpness is proceeding fast [11-14], direct control of the AFM tip will remain important. The proposed method to monitor the probing profile of the mounted tip can provide an important piece of information which can be incorporated routinely in the description and discussion of AFM experiments.

\footnotetext{
\#1 The range below $100 \mathrm{~nm}$ will be defined in the following as mesoscopic.
}

\section{Experimental}

AFM micrographs were recorded at ambient conditions with a Nanoscope 2 (Digital Instruments, Inc., Santa Barbara, CA, USA). The microscope was placed on a vibration-protective table in an acoustically isolated room. Commercial pyramidal $\mathrm{Si}_{3} \mathrm{~N}_{4}$ tips mounted on a $\mathrm{V}$-shape cantilever with a force constant of $0.06 \mathrm{~N} / \mathrm{m}$ (Nanoprobes ${ }^{\mathrm{TM}}$ ) were employed [14]. Supplementary measurements were done with a "super tip" (Fig. 1) which was prepared according to Keller and Chou [11].

Two imaging modes, "force" and "height", were used. The force mode (quasi-constant height) allows the evaluation of morphological details with higher contrast. The height mode (constant force) offers 3D surface profile. In the case of relatively soft polymer materials low force was kept down to $5 \mathrm{nN}$. High-modulus materials were scanned with $10-30 \mathrm{nN}$ forces. Image filtering was not used during scanning. All presented data were not processed except the UHMW-PE image which was low-pass filtered.

Typical artifacts were demonstrated by examples chosen from different projects which are presently under investigation at the University of Twente. Single crystals of $\mathrm{SrTiO}_{3}$ were supplied by Akzo International Research Single Crystal

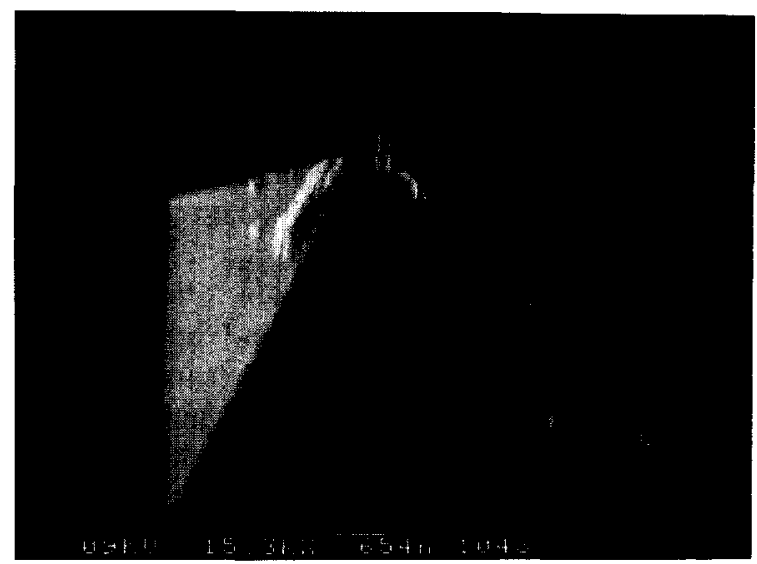

Fig 1. Scanning electron micrograph of a carbon tip grown on top of a commercial $\mathrm{Si}_{3} \mathrm{~N}_{4}$ tip. The electron beam was focused on the peak of the needle in hydrocarbon-contaminated vacuum. 
Technology, Ibbenbühren, Germany. The crystals had been cut parallel to the (305) orientation to obtain platelets of $10 \times 10 \times 1 \mathrm{~mm}$ and the (305) surface was mechanically-chemically polished.

For energetic reasons, the crystals possess a well defined, saw-tooth surface structure of small alternating (100) and (001) steps. After treating such a crystal for $10 \mathrm{~h}$ at $\sim 750^{\circ} \mathrm{C}$ in vacuum, small terraces are formed with a 3:5 width-toheight ratio. On average, the width corresponds to 10 unit cells $(4 \mathrm{~nm})$ and the height to 3-4 unit cells $(1-2 \mathrm{~nm})$. Annealing of the crystals at $1100^{\circ} \mathrm{C}$ for $20 \mathrm{~h}$ in flowing oxygen resulted in a rearrangement of the atoms in the surface and the formation of larger terraces, constructed from alternating (101) and (103) planes.

\section{Results and discussion}

Fig. 2 shows three typical examples of AFM artifacts monitored with commercial $\mathrm{Si}_{3} \mathrm{~N}_{4}$ tips [14]. The micrographs in Figs. $2 a$ and $2 b$ show a gold (111) surface on which a monolayer of a Högberg compound had been adsorbed via thiogroups [15]. According to the size of the absorbed molecules structural details should be in the range of 7-8 $\AA$. Both pictures were taken from the same sample using two different tips. The images do not show the actual surface structure but give an idea about distortions depending on the shape of the tip. The pictures demonstrate how sharp particles or protrusions on the sample surface can probe the surface of the tip and result in the incorporation of tip images at various places of the micrograph. The columnar or sheet structures in Fig. $2 b$ are rather unusual while the pyramidal artifacts in Fig. 2a are well known [6,7]. The image in Fig. $2 b$ can be explained assuming a blade-shaped tip. The artificial origin of these patterns can be recognized by the sharp demarcation, prominent appearance and the orientation coherent with the scan direction. Only the picture in Fig. 2a approaches the real situation and this only with regard to the areas in between the pyramidal elevations.

Fig. 2c shows an image of a polished stainless steel surface which had been sputtered with gold, so the steel surface is covered by small Au crystallites. In this case, artifacts are observed which do not posses an angular shape and orientation. The round elevations with small holes in the centre are not genuine. When the same surface was monitored with another, better tip, the structural details became smaller and the holes vanished. Considering the picture in Fig. $2 \mathrm{~d}$ as the more realistic, the delusive structure in Fig. $2 c$ may be explained by a twinned tip.

The distortion of the images in Figs. $2 e$ and $2 f$ is more difficult to be recognized because the AFM micrographs reproduce the true surface morphology qualitatively correct. The figures show the surface of a gel-drawn ultrahigh molecular weight polyethylene film. An UHMW-PE sample was crystallized from semidilute solution and after removal of the solvent drawn uniaxially to an extension of $k=15$. The nearly perfectly oriented fibrous sample shows a texture where the polyethylene chains are bundled to nanofibrils [16]. The observation of $50 \mathrm{~nm}$ wide strands in Fig. 2e (commercial $\mathrm{Si}_{3} \mathrm{~N}_{4}$ tip) is in agreement with other structural investigations $[16,17,20]$. However, it must be expected that the diameter of the nanofibrils is enlarged and that the real shape might be disguised by the convolution with the tip surface structure. When the same PE fibre was inspected by the "super tip" shown in Fig. 1, the observed diameter of the nanofibrils became smaller and imperfections of the fibrillated structure became more distinct (Fig. 2f).

While it is evident in the case of Figs. 2a and $2 b$ that the structural details cannot be real, unambiguous recognition of the image distortions in Figs. $2 c-2 f$ depends on the information about the profile of the probing tip.

Direct characterization or calibration of an AFM tip requires a gauge which fulfills the following conditions:

- The gauge should have a macroscopically regular surface with known atomic structure and well defined superstructure.

- The superstructure should contain atomically sharp elevations varying periodically within a few nanometers in $z$-direction.

- The surface must be rigid and invariant with time at ambient conditions. 

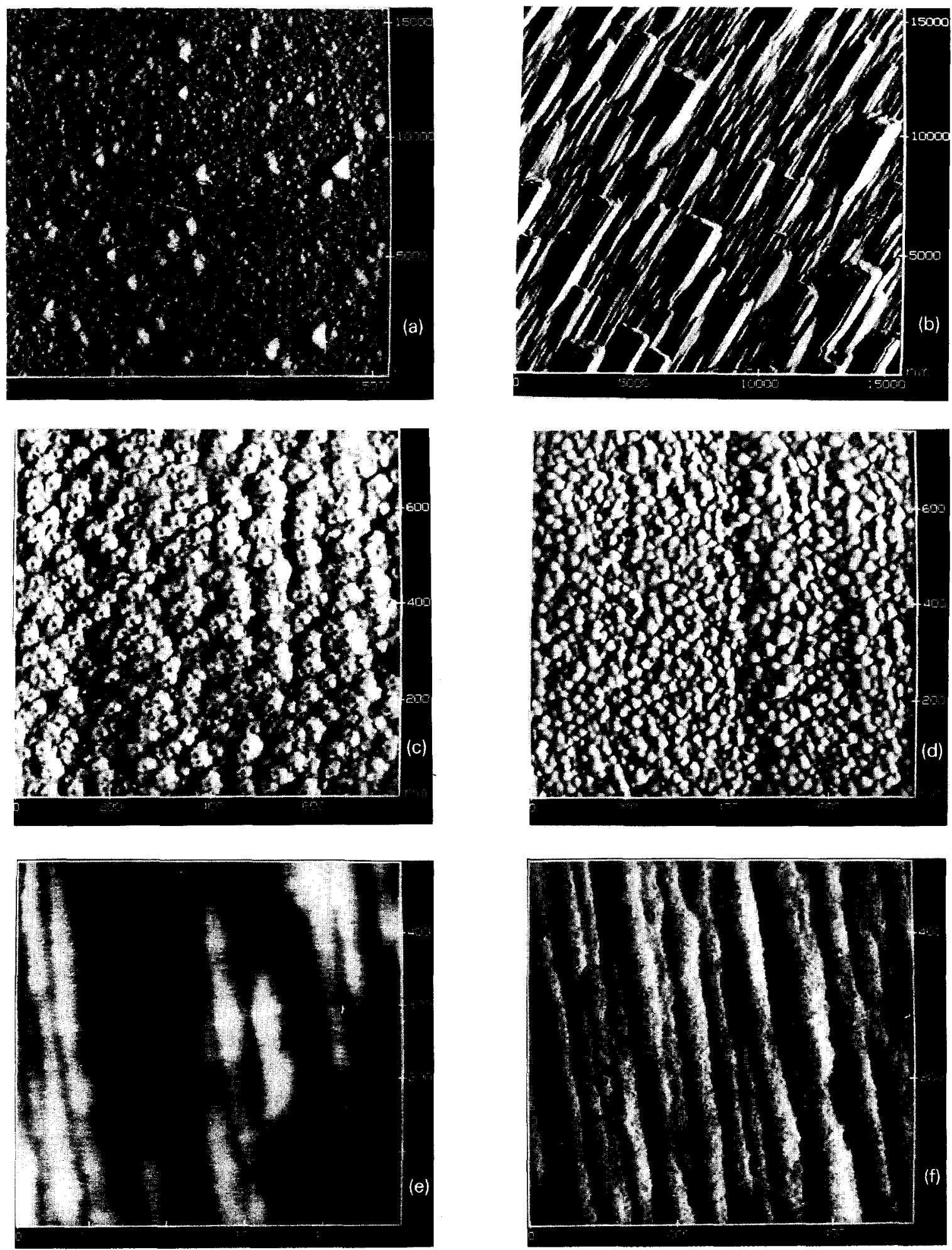

Fig 2. Selected artifacts recorded by atomic force microscopy. See text for a description. 
- In order to check the image distortions for a particular tip and probing mode in situ, recording of an image of such a test surface must be possible as a routine and fast operation before or after an unknown surface is monitored by AFM.

In the following we wish to demonstrate a test surface or gauge which fulfills these conditions rather perfectly. It is a specially treated surface of a perovskite-type crystal which was originally developed for epitaxial growth of high-temperature superconducting $\mathrm{YBa}_{2} \mathrm{Cu}_{3} \mathrm{O}_{x}$ [21]. By an etching/annealing procedure it was possible to obtain a surface with a regular saw tooth profile as shown in the transmission electron micrograph in Fig. 3. $\mathrm{SrTiO}_{3}$ crystals were etch-polished parallel to the (305) direction to become practically atomically flat. The resulting surface can be described as alternating very small (100) and (001) steps which are piled up from not more than a few atoms. When the crystal is annealed under vacuum the steps grow regulary as shown in Fig. 3. The annealing allowed the atoms to rearrange and a well defined indented topology was formed from inclined (101) and (103) surfaces. The TEM section through the surface reveals the rather perfect mesoscopic order with well defined angles between the lattice planes.

Fig. 4 shows a series of AFM images which were recorded from such a surface by different commercial $\mathrm{Si}_{3} \mathrm{~N}_{4}$ tips. The tips or needles were taken from different locations on the wafer from which they had been prepared. Obviously, most of the images do not represent real true-to-scale reproductions.

A nearly perfect surface image was obtained only with one tip selected out of 20 . Fig. $4 a$ represents a $3 \mathrm{D}$ lineplot of the grooved surface. The rather regular arrangement of parallel rib-

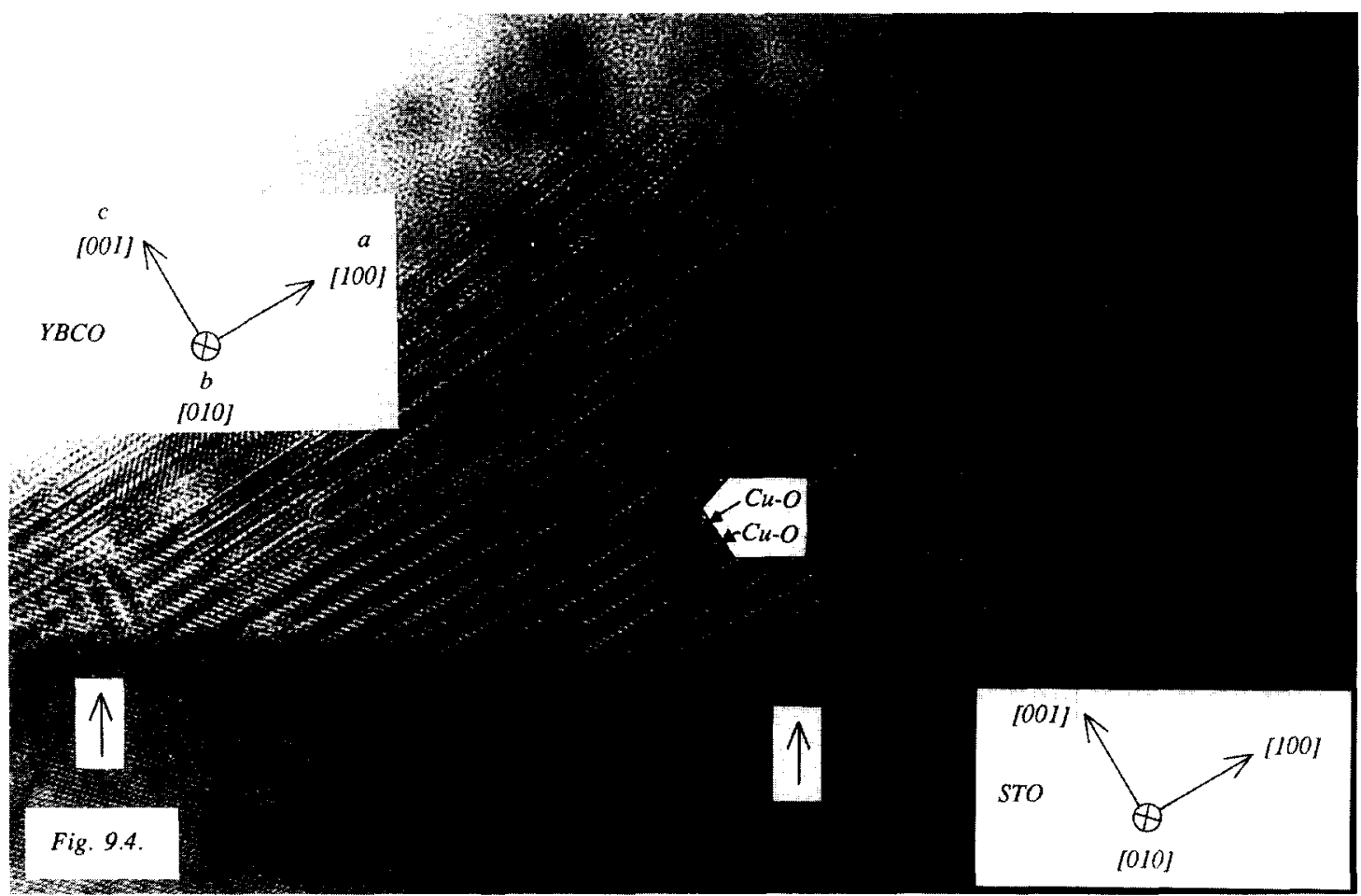

Fig 3. High-resolution transmission electron micrograph of a cross section through a $\mathrm{SrTiO}_{3}$ crystal with a sputtered film of $\mathrm{YBa}_{2} \mathrm{Cu}_{3} \mathrm{O}_{x}$. The section runs perpendicular to the (305) plane of $\mathrm{SrTiO}_{3}$. Exact crystallographic directions are indicated in the figure. The step structure is clearly visible at the interface between the $\mathrm{SrTiO}_{3}$ substrate and the HTC superconductor film. Sample was annealed at $750^{\circ} \mathrm{C}$ in vacuum before the superconductor film was grown on it. 

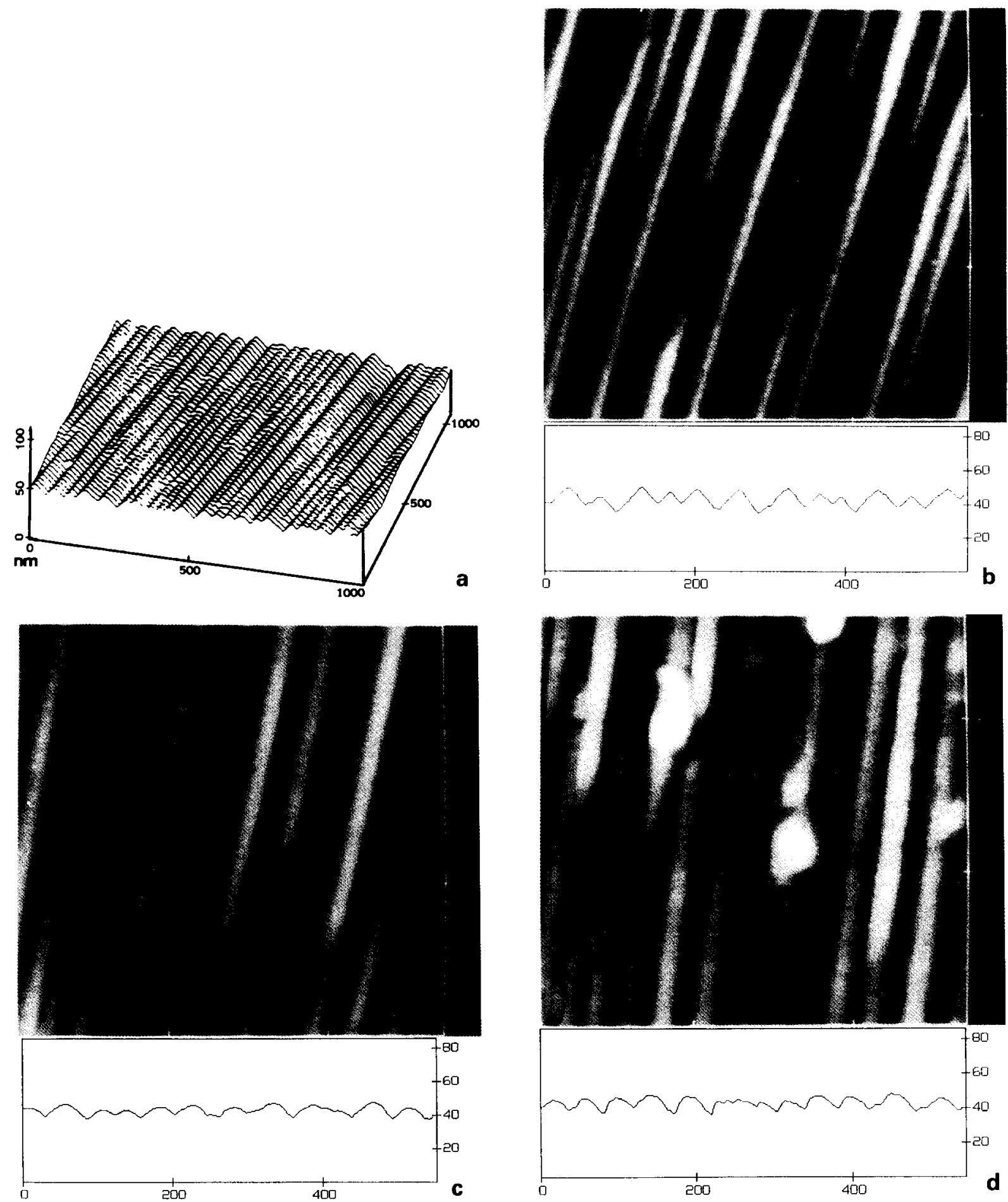

Fig 4. Series of AFM images and corresponding $2 \mathrm{D}$ profiles recorded by different commercial $\mathrm{Si}_{3} \mathrm{~N}_{4}$ tips from the same indented $\mathrm{SrTiO}_{3}$ surface from which an example is shown in Fig. 3. Only (a) and (b) give a good reproduction of the surface. The images become distorted past recognition as the deformation of the needle becomes more severe from (c) to (f). 

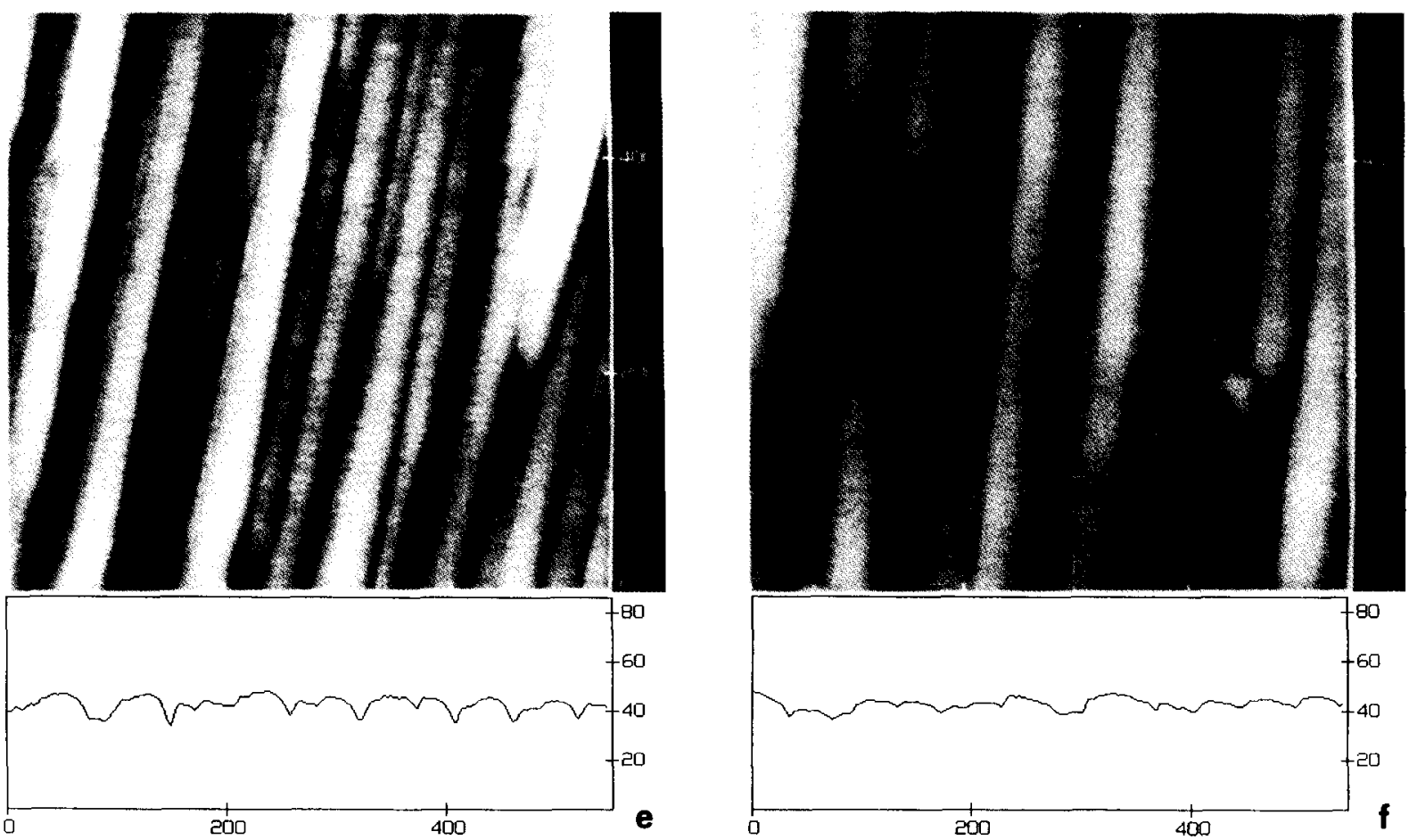

Fig. 4 (continued).

bons or stripes with alternating inclination is disturbed only by some undefined spot-like elevations which might be either impurities or defects from imperfect polishing. The enlargement in Fig. 4b shows clearly the true step structure of the $\mathrm{SrTiO}_{3}$ crystal with rather sharp and correct angles.

Fig. 5 gives an image of the same surface (Fig. 4b) monitored with a "super tip" (see Fig. 1). The step structure is observed correctly but the sharp edges were imaged with considerable smoothing. The radius of the curvature of the round edges in the image is directly related to the radius of the peak of the needle which can thus be evaluated to $10( \pm 2) \mathrm{nm}$.

Figs. 4c-4f show the same $\mathrm{SrTiO}_{3}$ surface recorded with less good $\mathrm{Si}_{3} \mathrm{~N}_{4}$ tips. The tip used for Fig. $4 c$ reproduced the shape of the steps strongly smoothed. Grooves were disguised due to the mutual convolution and multiple imaging. The picture imparts already a qualitatively wrong impression. In investigations on the pore struc- ture of micro- and ultrafiltration membranes, a similar distortion of the image resulted in total masking of the pores [22]. The radius of the peak of the needle can directly be estimated from Fig. $4 \mathrm{c}$ to be around $40 \mathrm{~nm}$.

Fig. 4d gives an image which was recorded with a tip which had a truncated pyramidal peak. Qualitatively, the corrugated structure is still reproduced and the occurrence of the particular elevations in the 2D image is real (see also Fig. 4b). However, the surface profile shows significant misrepresentation of the actual structure. Neither the dimensions nor the shape of the grooves are correctly reproduced. From the truncated peaks in the surface profile, it can be estimated that the peak of the needle is a flat plateau of roughly $15 \times 15 \mathrm{~nm}^{2}$.

Another example of a needle with a truncated peak is given in Fig. 4e. In this case, the needle is not only flattened but it possesses apparently several small protrusions which can be in contact with different points of the surface simultane- 


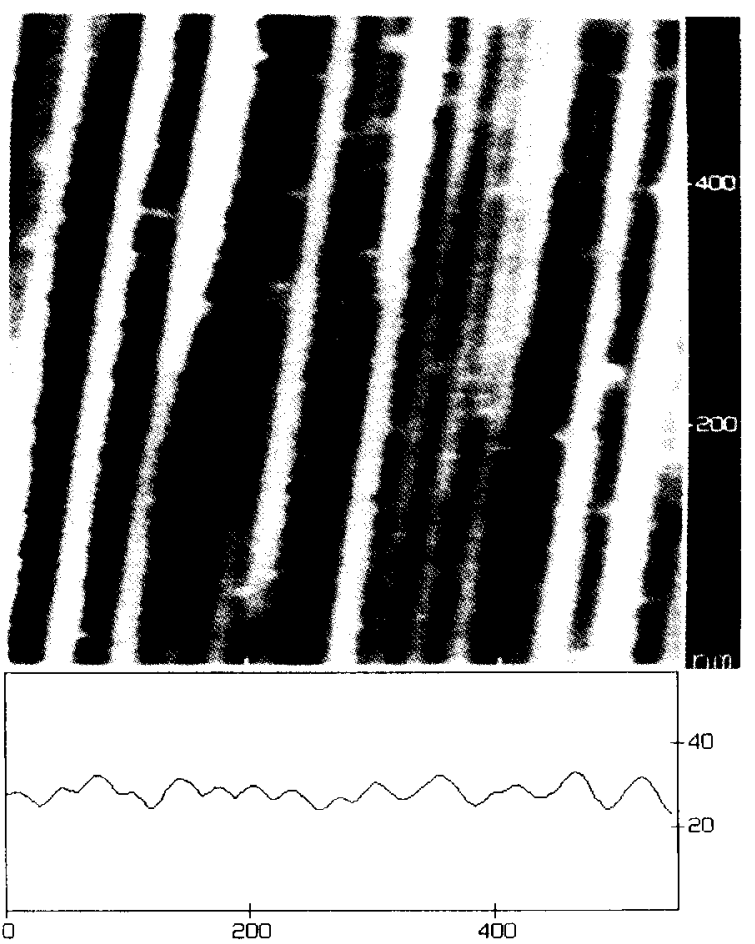

Fig 5. AFM image of the indented $\mathrm{SrTiO}_{3}$ surface recorded with the "super tip" shown in Fig. 1.

ously. A strongly distorted image results and the apparent profile does not resemble the actual topology. However, when the same needle was used to image a flat surface of a $\mathrm{NaCl}$ crystal at high resolution, the regular packing of the atoms in the surface was monitored faithfully and with excellent quality, as demonstrated by the atomic force micrograph in Fig. 6.

Another tip which is completely useless for mesoscopic surface characterization is demonstrated in Fig. 4f. Surface corrugations are masked, flattened and also their periodicity is strongly misrepresented. In this case it becomes even difficult to make any conclusions regarding the malformation of the tip. It is probably a blade-shaped tip, discussed above for the distorted image in Fig. $2 b$.

Fig. 7 demonstrates examples of images with atomic resolution where the selected tip from Fig. 4a was used. Due to the nearly perfect shape and the rigidity of the samples, the contact be- tween this tip and the surface can be highly localized and the basis for visualisation of single atomic details is rather optimal.

Fig. 7b shows small gold particles which were deposited on mica in vacuum at room temperature. Terraces from different (111) layers are observed even when their width was only $6 \AA$. Fig. 7a represents an image of a (111) gold surface on which $\mathrm{Zn}$-phthalocyanine molecules were adsorbed. In this case, the gold surface was prepared at elevated temperature $\left(250^{\circ} \mathrm{C}\right)$ in order to obtain large, atomically flat surfaces. It is demonstrated that the molecules are arranged in regular patterns within local domains with a diameter around $40 \mathrm{~nm}$.

Fig. 7c gives the image of a $\mathrm{TaS}_{2}$ crystal. The picture was not filtered or otherwise processed and could be reproduced repeatedly at the same location, independently of the scan direction. Several point defects can be observed which are marked by white arrows. In some cases these vacancies were reproduced in successive measurements and we believe that the image gives an example of real atomic resolution.

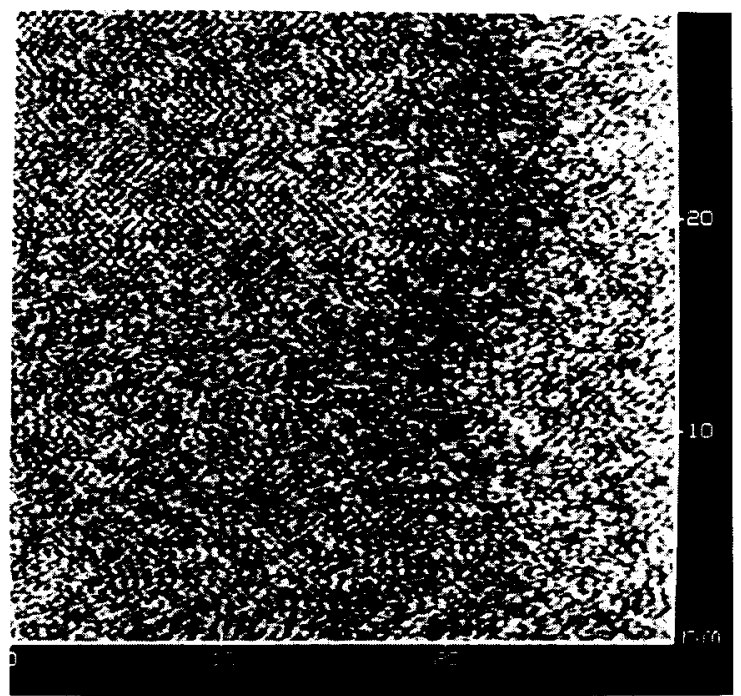

Fig 6. AFM image showing the (100) surface of a $\mathrm{NaCl}$ crystal. The image was recorded with the same needle as used for the picture in Fig. 4e. In spite of the truncated shape of the tip, the atomic structure of the $\mathrm{NaCl}$ surface is monitored with excellent resolution and little distortion. 

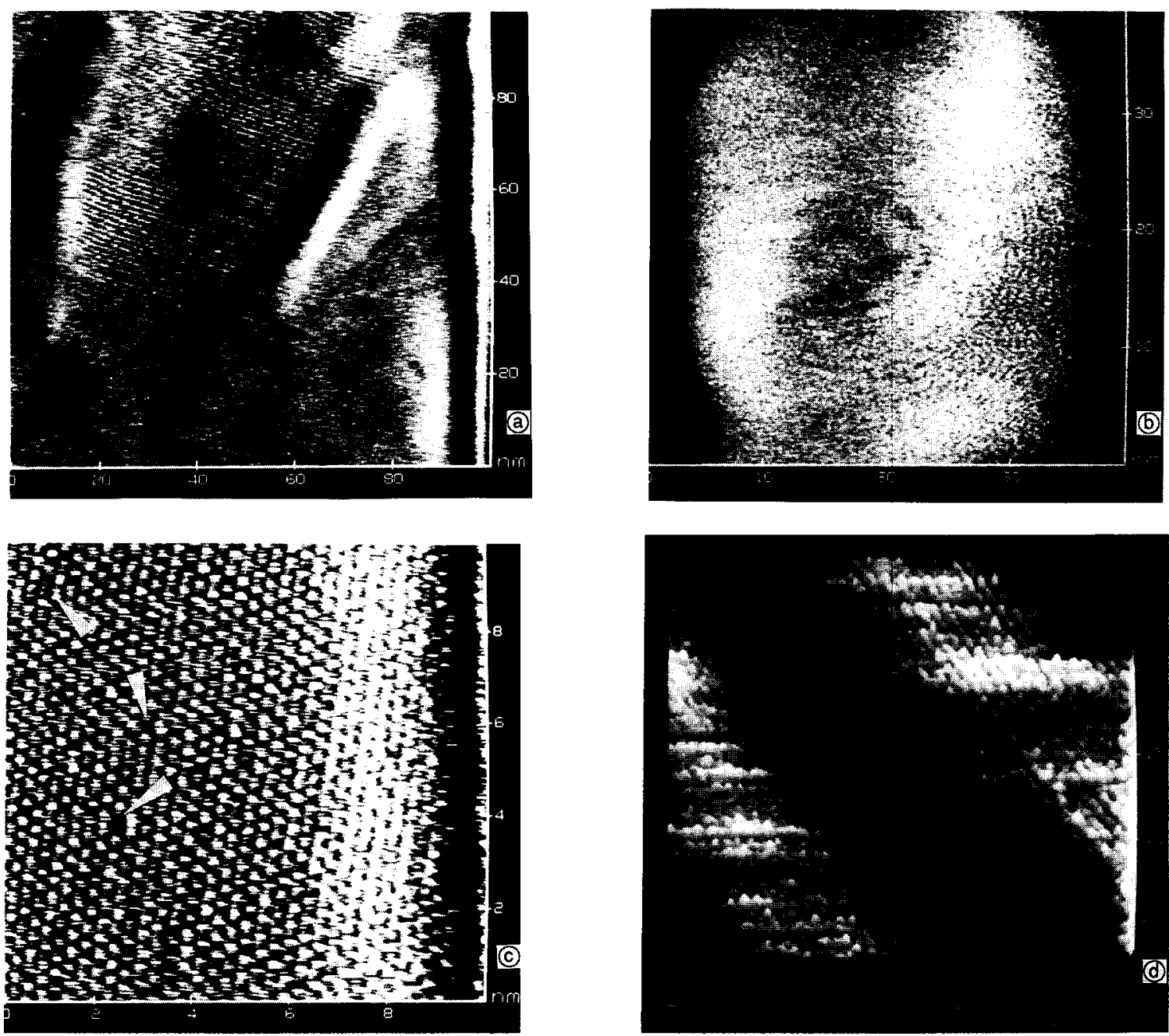

Fig 7. Selected examples of AFM images showing real near-atomic resolution. All images were recorded with a first class needle like the one used for Figs. 4a and 4b. (a) Image of a (111) gold surface on which Zn-phthalocyanine molecules were adsorbed in regular patterns within local domains. (b) Gold film sputtered at room temperature on glass; (111) terraces of a width of $6 \AA$ can still be resolved. (c) Image of a $\mathrm{TaS}_{2}$ crystal with several point defects which could be observed in successive measurements. (d) Surface image of gel-drawn UHMW-PE; individual polyethylene chains are resolved on the round shaped surfaces of two nanofibrils.

Finally, Fig. 7d demonstrates atomic resolution in the surface image of gel-drawn UHMW-PE as already discussed in the context of Figs. $2 \mathrm{e}$ and $2 \mathrm{f}$. The round-shaped surface of two nanofibrils are shown which have a diameter of $\sim 20 \mathrm{~nm}$ [16]. It appears that polyethylene chains are regularly aligned at the surface of the nanofibrils with periodicity about $5 \AA$.

\section{Conclusions}

The use of a gauge like the high-temperaturetreated (305) surface of a $\mathrm{SrTiO}_{3}$ crystal provides an import means of selecting perfect tips and evaluating possible image distortions. Imperfections are rather usual for the preparation of microfabricated AFM tips [6,14]. Often the growth 
of the tip is not terminated as a point-like peak but as a truncated pyramid with a top area of several up to hundreds of nanometers.

Although defective tips can give a rather perfect atomic resolution from flat crystal surfaces (Figs. 4e and 6), it must be realized that the atomic structure results from multiple contacts of the tip with the regular surface and can only represent the regular periodicity of the surface. As long as the different atoms in the surface are not probed independently, it cannot be expected that particular details like lattice defects become visible. Real atomic resolution can also not be expected for soft materials because of the elastic and inelastic deformation and the resulting increase of the contact area [10]. In the case of soft materials, the resolution appears to be limited to 2-3 nm. However, the images in Fig. 7 demonstrate the excellent resolution which can be achieved by commercial AFM from rigid surfaces, provided they are carefully selected. Real atomic resolution is approached at least very closely and it is shown that the atomic arrangement can be monitored in very small ordered domains.

\section{Acknowledgement}

The present work was funded by the "Onderzoek Stimulerings Fonds" of the University of Twente within the program "Nanoscale architectures".

\section{References}

[1] G. Binnig, C.F. Quate and C. Gerber, Phys. Rev. Lett. 56 (1986) 930.
[2] H. Heinzelmann, E. Meyer, P. Grütter, H. Rudin and H.-J. Güntherodt, in: Scanning Tunneling Microscopy and Related Methods, Eds. R.J. Behm et al. (Kluwer, Dordrecht, 1990) pp. 443-467.

[3] D. Rugar and P. Hansma, Phys. Today 43 (October 1990) 23.

[4] D. Sarid and V. Elings, J. Vac. Sci. Technol. B 9 (1991) 431.

[5] S.N. Magonov, S. Kempf, M. Kimmig and H.-J. Cantow, J. Polym. Sci. Polym. Phys. Ed. 26 (1991) 715.

[6] P. Grütter, W. Zimmermann-Edling and D. Brodbeck, Appl. Phys. Lett. 60 (1992) 2741.

[7] S.N. Magonov, A.Ya. Gorenberg and H.-J. Cantow, Polym. Bull. 28 (1992) 577.

[8] A.S. Lea, A. Pungar, V. Hlady, J.D. Andrade, J.N. Herron and E.W. Voss, Langmuir 8 (1992) 68.

[9] H.G. Dikland, S.S. Sheiko, L. van der Does, M. Möller and B. Bantjes, Polymer 34 (1993) 1773.

[10] T.M. Weihs, Z. Nawaz, S.P. Jarvis and J.B. Pethica, Appl. Phys. Lett. 59 (1991) 3536.

[11] D.J. Keller and Chou Chih-Chung, Surf. Sci. 268 (1992) 333.

[12] Ultralevers ${ }^{\mathrm{TM}}$, Park Scientific Instruments, Sunnyvale, CA 94089 , USA.

[13] O. Wolter, T. Bayer and J. Greschner, J. Vac. Sci. Technol. B 9 (1991) 1353.

[14] NanoProbes ${ }^{\mathrm{TM}}$, Digital Instruments Inc., Santa Barbara, CA 93117, USA.

[15] E.U. Thoden van Velzen, J.F.J. Engbersen and D.N. Reinhoudt, to be published.

[16] S.N. Magonov, S.S. Sheiko, R.A.C. Deblieck and M. Möller, Macromolecules 26 (1993) 1380.

[17] P.F. van Hutten, C.E. Koning and A.J. Pennings, Makromol. Chem. Rapid Commun. 4 (1983) 605.

[18] P. Smith and P.J. Lemstra, Makromol. Chem. 180 (1979) 2983;

P. Smith, P.J. Lemstra and H.C. Booij, J. Polym. Sci. Polym. Phys. Ed. 19 (1981) 877

[19] B.J. Palys, G.J. Puppels, D. van den Ham and F.D. Feil, J. Electroanal. Chem. 326 (1992) 105.

[20] J.M. Brady and E.L. Thomas, Polymer 30 (1989) 1615.

[21] W.A.M. Aarnink, E.M.C.M. Reuvekamp, M.A.J. Verhoeven, M.V. Pedyash, G.J. Gerritsma, A. van Silfhout and H. Rogalla, Appl. Phys. Lett. 61 (1992) 607.

[22] P. Dietz, P.K. Hansma, O. Inacker, H.-D. Lehmann and K.-H. Herrmann, J. Membr. Sci. 65 (1992) 101. 